\title{
Multivariable Analysis of Factors Affecting Length of Stay and Hospital Charges After Atlantoaxial Fusion
}

\author{
Jian Guan ${ }^{1}$, Michael Karsy ${ }^{1}$, Meic Schmidt ${ }^{1}$, Andrew T. Dailey ${ }^{1}$, Erica Bisson ${ }^{1}$ \\ 1. Department of Neurosurgery, University of Utah
}

$\square$ Corresponding author: Erica Bisson, erica.bisson@hsc.utah.edu

Disclosures can be found in Additional Information at the end of the article

\section{Abstract}

\section{Background}

Atlantoaxial fusion is an effective procedure for treating degenerative, traumatic, and congenital abnormalities that result in upper cervical instability; however, data on which factors affect the length of stay and hospitalization-related charges are limited. The purpose of this study was to evaluate the pre-, intra-, and postoperative variables that affect these healthcare cost factors for patients undergoing posterior atlantoaxial fusion.

\section{Methods}

We retrospectively identified from a clinical database 59 patients who underwent isolated posterior atlantoaxial fusion at a single institution from 2010 to 2015. Demographic, clinical, and surgical variables from a clinical database were analyzed with respect to the length of hospital stay and hospital charges. T-test and Chi-square testing, as well as univariate and multivariable analysis, were performed with $\mathrm{p}<0.05$ considered significant.

\section{Results}

On multivariable analysis, a variety of preoperative, intraoperative, and postoperative variables were associated with prolonged hospitalization and higher hospital charges, including tobacco use, preoperative diagnosis, operating room time, and the need for intraoperative blood transfusion.

\section{Conclusions}

Varied preoperative, intraoperative, and postoperative factors are associated with increased length of hospitalization and higher hospital charges after atlantoaxial fusion. Familiarity with

Received 03/20/2017 Review began 04/12/2017 Review ended 04/13/2017 Published 04/18/2017

C) Copyright 2017

Guan et al. This is an open access article distributed under the terms of the Creative Commons Attribution License CC-BY 3.0., which permits unrestricted use, distribution, and reproduction in any medium, provided the original author and source are credited.
Categories: Neurosurgery

Keywords: atlantoaxial fusion, multivariable analysis, length of stay, hospital charges, comorbidities

\section{Introduction}

Because of its complex anatomy, the atlantoaxial region is uniquely vulnerable to certain degenerative, traumatic, and neoplastic pathological conditions [1]. Posterior cervical fusion is one of the multiple surgical options that have allowed for a significant reduction in the need for 
external bracing using "halo" systems [2]. Although fusion rates after posterior cervical fusion range from $93 \%$ to $99 \%$ [3] and rates of complications are low (2\%-4\%) [4], there is a lack of information regarding what factors influence the length of hospitalization itself [5]. An understanding of such influences is becoming increasingly important as surgical care becomes more outcomes focused [6], and measures, such as length of stay (LoS), are associated with the incidence of more serious complications, such as postoperative infection [7] and thrombotic events [8]. In addition, LoS is thought to be a major driver of health care costs, a growing cause of concern among healthcare providers and policy makers alike [9]. As value-based care gains more traction, careful attention to hospital charges and what can be done to minimize them, especially those associated with expensive surgical interventions, becomes increasingly valuable [10]. In this study, we examined various pre-, intra-, and postoperative factors that may be associated with LoS and hospital charges following posterior atlantoaxial fusion.

\section{Materials And Methods}

\section{Study population}

After obtaining approval from the University of Utah Institutional Review Board with a waiver of informed consent, we queried a clinical database to obtain information about patients 18 years and older who underwent C1-C2 posterior spinal fusion between May 1, 2010, and April 30, 2015. This information was then confirmed and supplemented by individual chart review. Patients were excluded if other procedures (subaxial fusion, laminectomy, etc.) were performed during the same procedure or hospitalization. All surgical procedures were performed by one of five experienced spine surgeons.

\section{Data collection}

Demographic, intraoperative, and postoperative information were collected on all patients. Demographic data included age, sex, body mass index (BMI), American Society of Anesthesiologists (ASA) Physical Status Classification System Grade, active tobacco use, active alcohol use, preoperative opioid use, employment status, marriage status, and insurance type. Relevant medical history including the presence of rheumatoid arthritis, Type 2 diabetes, hypertension, pulmonary comorbidities (i.e., asthma, chronic obstructive pulmonary disease, pulmonary embolism, bronchitis), cardiac comorbidities (i.e., atrial fibrillation, heart murmur, arrhythmia, myocardial infarction, coronary artery disease, congestive heart failure, mitral valve prolapse), previous cervical spine surgeries, and the presence of polytrauma was also obtained. Preoperative diagnoses were recorded as trauma (e.g., Jefferson fractures, odontoid fractures), instability (due to rheumatoid arthritis or congenital causes), or degenerative disease (e.g., symptomatic facet arthropathy).

Intraoperative variables included surgical time, estimated blood loss, intraoperative blood transfusion, intraoperative crystalloid and colloid infusion volumes, intraoperative complications, and axial instrumentation type. Postoperative variables consisted of the need for readmission within 30 days, postoperative complications, and discharge destination. Discharge destinations included home, skilled nursing facility, acute rehabilitation facility, and psychiatric facility, which were subsequently dichotomized to those discharged to home and to other destinations. Finally, charge data for each hospitalization was obtained.

\section{Statistical analysis}

Continuous variables in all cases were analyzed utilizing Student's t-test, while categorical variables were analyzed utilizing Chi-squared analysis. We performed both univariate and multivariable analysis for LoS and hospital charges. For univariate analysis of LoS, we dichotomized hospitalization length into "extended" ( $>4$ days) and "normal" ( $\leqslant 4$ days) based on hospitalizations longer than the 75th percentile in keeping with previous studies [11]. For 


\section{Cureus}

multivariable analysis, LoS was examined as a continuous variable. We utilized a stepwise regression analysis to construct our model, with a threshold $\mathrm{p}<0.20$ for inclusion and a $\mathrm{p}<0.05$ being defined as statistically significant in our final model. For univariate analysis of hospital charges, we dichotomized charges into "high" (> \$62,200) and "normal" $(\leqslant \$ 62,200)$ based on charges greater than the 75th percentile. For multivariable analysis, hospital charges were examined as a continuous variable. We utilized a stepwise regression analysis to construct our model, with a threshold $\mathrm{p}<0.20$ for inclusion and a $\mathrm{p}<0.05$ being defined as statistically significant in our final model.

All statistical analysis was performed using SPSS V20.0 (IBM Corp., Armonk, NY). The Strengthening the Reporting of Observational Studies in Epidemiology (STROBE) guidelines were used during the preparation of this work [12].

\section{Results}

\section{Length of stay}

Among the 59 patients who met our inclusion and exclusion criteria, mean LoS was 4.2 days (95\% confidence interval of 2.94 to 5.5 days). For our univariate analysis, 13 patients met the criterion for "extended" LoS with an average LoS of 9.2 days and 46 met the criterion for "normal” LoS with an average LoS of 2.8 days. Demographic factors were largely similar between the normal and extended groups (Table 1), including age, BMI, sex, marital status, insurance status, and employment status. ASA grade was significantly higher in the extended stay group ( $p=0.026)$; patients in the normal LoS group were less likely to be on opioids preoperatively ( $15 \%$ vs $48 \%, \mathrm{p}=0.036$ ), although other comorbidities were not statistically different between normal and extended LoS patients. There was no significant difference between the two groups in preoperative surgical diagnosis. The rate of polytrauma did not differ between the groups (13\% extended LoS vs. 6\% normal LoS, $p=0.602$ ).

\begin{tabular}{|c|c|c|c|c|}
\hline & & Extended $(\mathrm{N}=13)$ & Normal (N=46) & p value* \\
\hline \multicolumn{2}{|l|}{ No. of female patients (\%) } & $6(46)$ & $25(54)$ & 0.601 \\
\hline \multicolumn{2}{|l|}{ Mean age in years \pm SD } & $63 \pm 19.1$ & $59 \pm 18.5$ & 0.446 \\
\hline \multicolumn{2}{|l|}{$\mathrm{BMI} \pm \mathrm{SD}$} & $27 \pm 9.4$ & $27 \pm 6.0$ & 0.851 \\
\hline \multicolumn{2}{|l|}{ Married (\%) } & 9 (69) & $29(63)$ & 0.681 \\
\hline \multicolumn{2}{|l|}{ Private insurance (\%) } & $4(31)$ & $20(44)$ & 0.410 \\
\hline \multirow{4}{*}{ Employment status (\%) } & Unemployed & $3(23)$ & $15(33)$ & \multirow{4}{*}{0.768} \\
\hline & Employed & $3(23)$ & $12(26)$ & \\
\hline & Retired & 7 (54) & $18(39)$ & \\
\hline & Student & $0(0)$ & $1(2)$ & \\
\hline \multirow{2}{*}{ ASA category (\%) } & Low (1-2) & $4(31)$ & $30(65)$ & \multirow{2}{*}{0.026} \\
\hline & High (3-5) & 9 (69) & $16(35)$ & \\
\hline \multicolumn{2}{|l|}{ Tobacco use (\%) } & 2 (15) & $3(7)$ & 0.311 \\
\hline \multicolumn{2}{|l|}{ Alcohol use (\%) } & $7(54)$ & $16(35)$ & 0.213 \\
\hline
\end{tabular}




\section{Cureus}

\begin{tabular}{|c|c|c|c|c|}
\hline \multicolumn{2}{|c|}{ Preoperative opioid use (\%) } & $2(15)$ & $22(48)$ & 0.036 \\
\hline \multirow{3}{*}{ Diagnostic category (\%) } & Trauma & $8(62)$ & $16(35)$ & 0.216 \\
\hline & Degenerative & 2 (15) & $14(23)$ & \\
\hline & Instability & $3(23)$ & $16(35)$ & \\
\hline \multicolumn{2}{|l|}{ Rheumatoid arthritis (\%) } & $0(0)$ & $9(20)$ & 0.083 \\
\hline \multicolumn{2}{|l|}{ Type 2 diabetes (\%) } & $5(39)$ & $8(17)$ & 0.106 \\
\hline \multicolumn{2}{|l|}{ Hypertension (\%) } & $3(23)$ & $11(24)$ & 0.950 \\
\hline \multicolumn{2}{|c|}{ Pulmonary comorbidity (\%) } & $4(31)$ & $13(28)$ & 0.860 \\
\hline \multicolumn{2}{|l|}{ Cardiac comorbidity (\%) } & $4(31)$ & $13(28)$ & 0.860 \\
\hline \multicolumn{2}{|c|}{ Previous cervical spine surgery (\%) } & $2(15)$ & $10(22)$ & 0.615 \\
\hline \multicolumn{2}{|l|}{ Polytrauma (\%) } & $1(13)$ & $1(6)$ & 0.602 \\
\hline \multicolumn{2}{|c|}{ Estimated blood loss $(\mathrm{ml}) \pm \mathrm{SD}$} & $263.9 \pm 182.8$ & $243.6 \pm 266.0$ & 0.798 \\
\hline \multicolumn{2}{|c|}{ Intraoperative crystalloid volume $(\mathrm{ml}) \pm \mathrm{SD}$} & $1931 \pm 773.9$ & $2415 \pm 943.0$ & 0.096 \\
\hline \multicolumn{2}{|c|}{ Intraoperative colloid volume $(\mathrm{ml}) \pm S D$} & $38.5 \pm 138.7$ & $76.1 \pm 234.9$ & 0.585 \\
\hline \multicolumn{2}{|l|}{ Room time (minutes) $\pm \mathrm{SD}$} & $280.1 \pm 82.6$ & $328.5 \pm 108.9$ & 0.143 \\
\hline \multicolumn{2}{|c|}{ Need for intraoperative blood transfusion (\%) } & $1(8)$ & $1(2)$ & 0.332 \\
\hline \multicolumn{2}{|c|}{ Intraoperative complications (\%) } & $3(23)$ & $0(0)$ & 0.001 \\
\hline \multirow{5}{*}{ Axial screw type } & Laminar & $0(0)$ & 7 (15) & 0.056 \\
\hline & Pedicle & 2 (15) & $3(7)$ & \\
\hline & Pars & $10(77)$ & $19(41)$ & \\
\hline & Transarticular & $1(8)$ & $4(9)$ & \\
\hline & Combination & $0(0)$ & $13(28)$ & \\
\hline \multicolumn{2}{|c|}{ Postoperative complications (\%) } & $3(23)$ & $2(4)$ & 0.032 \\
\hline \multicolumn{2}{|l|}{ 30-day readmission (\%) } & $1(8)$ & $1(2)$ & 0.332 \\
\hline \multicolumn{2}{|l|}{ Hospital charges \pm SD } & $\$ 97,867.23 \pm 87,665.70$ & $\$ 49,825.51 \pm 18.795 .21$ & 0.001 \\
\hline \multirow{2}{*}{ Discharge destination } & Home & $6(46)$ & $37(80)$ & 0.014 \\
\hline & Other & $7(54)$ & $9(20)$ & \\
\hline
\end{tabular}

\section{TABLE 1: Univariate Analysis for Extended Versus Normal Length of Stay Groups}

SD: standard deviation; BMI: body mass index; ASA: American Society of Anesthesiologists

${ }^{*} \mathrm{P}$ value $<0.05$ was considered significant. 


\section{Cureus}

Intraoperatively, patients in the extended stay group were more likely to have experienced complications $(23 \%$ vs. $0 \%, p=0.001)$. A total of three intraoperative complications were noted -two dural tears and one vertebral artery injury-resulting in an overall intraoperative complication rate of $5.1 \%$. Other intraoperative factors, including estimated blood loss, volume of crystalloid or colloid infused, operative room time, need for blood transfusion, and axial screw type, were not significantly different between the two groups, although there was a trend towards laminar screws in the normal LoS group and pars screws in the extended LoS group $(\mathrm{p}=$ 0.056).

Postoperatively, patients in the normal LoS group were significantly more likely to be discharged home than those in the extended LoS group $(p=0.001)$. There were five postoperative complications-one wound infection requiring washout, one patient with anemia requiring blood transfusion, one patient who developed supraventricular tachycardia, one case of intravenous infiltration requiring operative debridement, and one aspiration event requiring reintubation-resulting in an overall rate of $8.5 \%$. Patients in the extended LoS group were significantly more likely to suffer from postoperative complications than the normal LoS group (23\% vs $4 \%, \mathrm{p}=0.032)$.

Utilizing stepwise regression to identify predictive factors for our multivariable model, we found polytrauma, discharge to a location other than home, tobacco use, and the need for intraoperative blood transfusion were associated with a significantly longer length of stay, while higher intraoperative crystalloid volumes and the presence of pulmonary comorbidities were associated with a significantly shorter length of stay (Table 2).

\begin{tabular}{|c|c|c|c|}
\hline Variable & $\beta$ coefficient & t statistic & p value* \\
\hline Discharge destination & 3.807 & 4.036 & $<0.001$ \\
\hline Polytrauma & 13.052 & 5.500 & $<0.001$ \\
\hline Tobacco use & 6.160 & 4.002 & $<0.001$ \\
\hline Crystalloid volume & -0.001 & -2.290 & 0.026 \\
\hline Intraoperative blood transfusion & 5.946 & 2.624 & 0.011 \\
\hline Pulmonary comorbidity & -2.040 & -2.170 & 0.035 \\
\hline
\end{tabular}

\section{TABLE 2: Multivariable Analysis of Factors Influencing Length of Stay}

*P value $<0.05$ was considered significant

Average charges for the extended LoS group were significantly higher than that for the normal LoS group $(\$ 109,878.15,95 \% \mathrm{CI} \pm \$ 46,235.78$ vs. $\$ 49,825.51,95 \% \mathrm{CI} \pm \$ 2,505.94, \mathrm{p}<0.001)$. Hospital charge data were not included in our multivariable analysis of LoS as we believe it unlikely that charges, tallied after discharge, could have any causal effect with prolonged hospitalization. 


\section{Cureus}

\section{Hospital charges}

The mean hospital charge for our patient cohort was $\$ 60,410.98$ (95\% confidence interval of $\$ 48,002$ to $\$ 72,820$ ). For our univariate analysis, 14 patients met the criterion for "high" hospital charges with an average value of $\$ 109,878.15$, and 45 met the criterion for "normal" hospital charges with an average value of \$45,021.19. Demographic factors were largely similar between the high and normal hospital charge groups (Table 3), although BMI was significantly lower in the high-charge group $(23.5 \pm 4.2 \mathrm{vs.} 27.8 \pm 7.1, \mathrm{p}=0.035)$. The rates of most major comorbidities were similar in the high- and normal-charge groups, but the high-charge group had a higher rate of tobacco use $(21 \%$ vs $4 \%, \mathrm{p}=0.046)$ and cardiac comorbidities ( $57 \%$ vs $20 \%$, $\mathrm{p}=0.007)$. Patients in the high-charge group were also much more likely to have surgery for a traumatic cause rather than instability or degenerative disease (86\% vs $27 \%$ ).

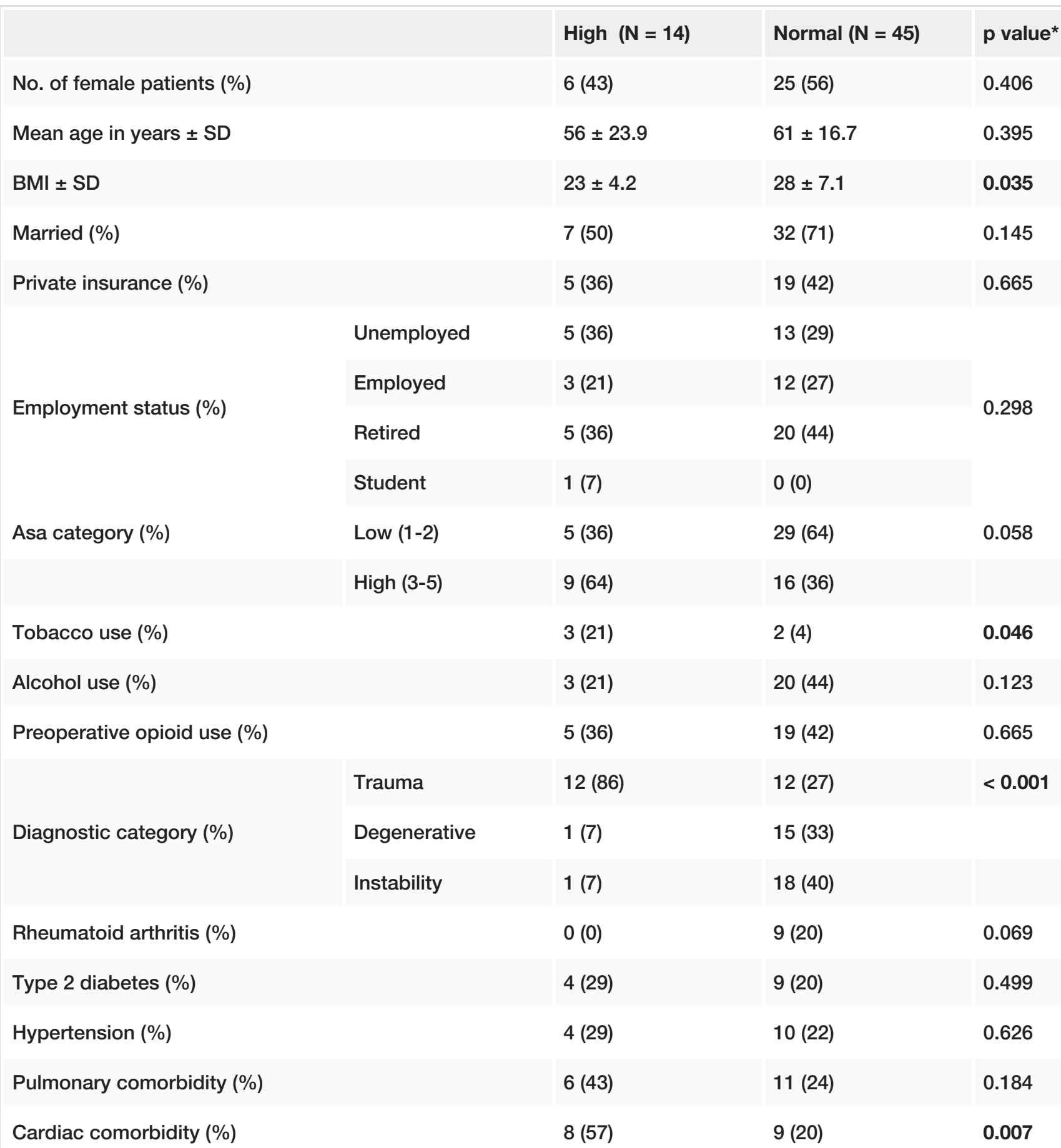




\section{Cureus}

Previous cervical spine surgery (\%)

Polytrauma (\%)

Estimated blood loss $(\mathrm{mL}) \pm \mathrm{SD}$

Intraoperative crystalloid volume $(\mathrm{mL}) \pm S D$

Intraoperative colloid volume $(\mathrm{mL}) \pm \mathrm{SD}$

Room time (minutes) \pm SD

Need for intraoperative blood transfusion (\%)

Intraoperative complications (\%)

Axial screw type

Length of stay

Pedicle

Pars

Postoperative complications (\%)

30-day readmission (\%)

Home

Discharge destination

Other
4 (29)

1 (7)

$335.6 \pm 138.3$

$2592.9 \pm 1105.6$

$107.1 \pm 289.5$

$312.3 \pm 93.5$

2 (14)

1 (7)

$1(7)$

$0(0)$

$8(18)$

0.381

(2)

0.374

$312.3 \pm 93.5$

0.472

$2220 \pm 855.6$

0.190

$55.6 \pm 191.4$

0.442

$335.6 \pm 138.3$

0.472

$0(0)$

0.010

$2(4)$

0.688

6 (13)

0.257

$10(71)$

19 (42)

Transarticular

$0(0)$

5 (11)

Combination

3 (21)

$10(22)$

$3(21)$

2 (4)

0.046

1 (7)

1 (2)

0.374

$6(43)$

37 (82)

$8(57)$

8 (18)

7 (50)

39 (87)

7 (50)

6 (13)

\section{TABLE 3: Univariate Analysis for High Versus Normal Hospital Charge Groups}

SD: standard deviation; BMI: body mass index; ASA: American Society of Anesthesiologists

${ }^{*} \mathrm{P}$ value $<0.05$ was considered significant.

Intraoperatively, patients in the high-charge group were more likely to have had a blood transfusion $(14 \%$ vs $0 \%, p=0.01)$. Other intraoperative factors were similar between the two groups. Postoperatively, patients in the high-charge group were significantly more likely to be discharged to a location other than home $(\mathrm{p}=0.004)$ and to experience postoperative complications $(p=0.046)$. Utilizing the same dichotomization scheme as described above, patients in the high-charge group were also significantly more likely to fall into the extended LoS category $(\mathrm{p}=0.004)$

Utilizing stepwise regression to identify predictive factors for our multivariable model, we found prolonged LoS, a preoperative diagnosis of trauma, longer operative time, and postoperative complications were associated with a significantly higher hospital charges, while 


\section{Cureus}

patients who required readmission within 30 days had significantly lower initial hospital charges (Table 4).

\begin{tabular}{|c|c|c|c|}
\hline Variable & $\beta$ coefficient & t statistic & p value* \\
\hline Length of stay & 8647.35 & 15.935 & $<0.001$ \\
\hline Diagnostic category & 6678.21 & 2.154 & 0.036 \\
\hline Operative time & 77.50 & 3.105 & 0.003 \\
\hline 30-day readmission & -63962.10 & -3.515 & 0.001 \\
\hline Postoperative complication & 31934.18 & 2.705 & 0.009 \\
\hline
\end{tabular}

\section{TABLE 4: Multivariable Analysis of Factors Influencing Hospital Charges}

${ }^{*} \mathrm{P}$ value $<0.05$ was considered significant

\section{Discussion}

The length of stay is a critical care measure after spine surgery for several reasons, ranging from its association with postoperative complications to increases in costs. Patients frequently ask how long they will be hospitalized after their operation, but this question is often difficult to answer, and evidence suggests that different factors play a role in determining the length of stay depending on what surgery is performed [5, 13]. From an economic point of view, patients in our extended stay group accrued an average of nearly $\$ 50,000$ more in hospital charges compared with those in the normal LoS group. With renewed focus on value in healthcare, determination of what factors may influence LoS becomes critical. For similar reasons, hospital charge drivers will likely become a steadily increasing area of interest in the next several years, with high-cost surgical interventions being the most heavily scrutinized.

In our study, preoperative factors associated with longer LoS on multivariable analysis included tobacco use and the presence of polytrauma. Tobacco use is associated with a wide array of surgical morbidities and mortality [14-16], including increased rates of intubation, infection, and myocardial infarction [17]. All of these complications frequently result in the need for extended hospitalization, and therefore, this relationship is unsurprising. This association, combined with evidence that preoperative [16] and even postoperative [18] smoking cessation can have benefits in patients undergoing spinal procedures, should prompt aggressive patient education on the hazards of tobacco use in relation to surgery. Patients experiencing polytrauma often require hospitalization for a variety of reasons and may undergo multiple surgical interventions. The extent of injury in these patients [19] combined with frequently higher rehabilitation needs [20] likely combine to necessitate longer hospitalizations.

Interestingly, the only preoperative variable associated with a shorter length of stay was the presence of a pulmonary comorbidity. Although preoperative pulmonary dysfunction is frequently a concern in patients, it is possible that, because of its distance from the diaphragm, cervical procedures such as atlantoaxial fusion may be uniquely less susceptible to the impact of such comorbidities [21]. This fact, combined with a higher degree of vigilance in these patients in relation to postoperative mobilization and incentive spirometry use, may combine to result in a paradoxical reduction in pulmonary-related complications and thus decreased 
LoS.

Intraoperatively, the need for blood transfusion and lower crystalloid infusion volumes were associated with longer LoS. Evidence suggests that under-resuscitation may lead to higher rates of complications following surgical intervention [22-23]. It is possible that patients who lose enough blood intraoperatively to require transfusion and those who receive less crystalloid are at higher risk for falling into this "under-resuscitated" category. This finding suggests that surgeons and anesthesiologists should be vigilant during surgery of the possibility of underresuscitation and correct any evidence of significant hypovolemia or anemia aggressively.

Postoperatively, inability to discharge home was associated with a significantly longer length of hospitalization. This is in keeping with previous reports of factors that are associated with longer LoS [5]. There are several possible explanations for this relationship. One is that patients who are unable to be discharged home are simply more ill and therefore require a longer time to recover following surgery. Another is that the time it takes to arrange discharge to a facility or rehabilitation unit may itself contribute to prolonged hospitalization. Regardless, careful disposition planning in these patients preoperatively-including discussions with the patient and the patient's caregivers-is likely prudent. Postoperative complications were also associated with increased length of stay. While this could be explained simply by the need for hospitalization to manage complications that arise (the aspiration event requiring reintubation), it is also possible that a longer hospital stay may itself lead to complications. For instance, had the patient with intravenous infiltration been discharged earlier and the intravenous line removed, this incident would have been avoided. This further highlights the benefits of timely discharge to avoid possible events related to the hospitalization itself.

In relation to charges, patients with a preoperative diagnosis of trauma were more likely to incur higher charges during their hospital stay. This is possibly related to the higher likelihood of an unplanned/ emergent surgery in these patients and the inherent costs therein [24]. Patients with cardiac comorbidities and those who were active tobacco users were also more likely to fall into the higher charge group. One possible explanation for this is an increased severity of illness allowing for a higher complication/comorbidity tier to be billed for the hospitalization. Unfortunately, our charge data are not granular enough to definitively show such differences, although this would be an interesting avenue for further study. Intraoperatively, longer operative times were associated with higher hospital charges, likely related to the direct costs of time in the operating room itself.

Postoperatively, LoS and postoperative complications were both associated with increased hospital charges. Charges for room and board, nursing, medications, and other fees are the likeliest explanation of the relationship between LoS and hospital charges. The higher charges associated with postoperative complications likely result from the resources needed to address these complications-return to the operating room, subspecialist consultations, etc.

Surprisingly, patients who required readmission within 30 days had significantly lower hospital charges compared with those who did not. One possible explanation for this is that these patients returned home earlier, thus incurring lower charges, but were subsequently readmitted because of issues related to such rapid discharge.

Modern techniques for atlantoaxial fixation have shown dramatic improvements in rates of fusion compared with the early, wiring-based approaches in both adults [25] and children [26]. Complication rates for these procedures are also low [27], although intimate knowledge of the local anatomy is paramount [28-29]. Despite these technical advances, data on patient and medical utilization-related outcomes remain scant. With healthcare-related costs coming into sharper focus in the past several years, the need to better define what factors influence cost drivers, such as duration of hospitalization and overall hospital charges, is becoming 
increasingly critical. We report one of the largest series of C1-C2 fusions at a single institution in the literature to date and the largest examining LoS and hospital charges as they relate to preoperative, intraoperative, and postoperative factors.

Our study has several limitations. The study is retrospective, and the sample size, while large for investigations of atlantoaxial fusion in the current literature, remains fairly limited. It is possible that some of the variables that were significantly associated with LoS on univariate analysis would have been found to be significant on multivariable analysis with a larger cohort, although this also suggests that the effect size would also be smaller. We hope that our findings help to guide further inquiries into factors that may be associated with higher hospital charges and increased LoS in this population, preferably utilizing data from multiple centers and significantly larger patient cohorts. Our study was limited to patients who received only isolated atlantoaxial fusions; although this likely reduced the influence of confounding factors, it also reduces generalizability to patients who require additional interventions.

Finally, our study examined charge data, a measure that, despite having some utility as a proxy for care costs [30], is not a substitute for actual cost data. The utility of charge data also varies based on the nature of payment for care, with a greater relevance in environments where "perdiem" and cash-for-service models are common versus those in which diagnosis-related group reimbursement is the norm. This fact also reduces the generalizability of our findings.

\section{Conclusions}

Posterior atlantoaxial fusion is a well-tolerated procedure with a low rate of complications. Various preoperative, intraoperative, and postoperative factors play a role in determining which patients will require a prolonged hospitalization and accrue higher hospital charges during their stay. Familiarity with these variables may help guide preoperative planning and patient counseling in those undergoing atlantoaxial fusion.

\section{Additional Information}

\section{Disclosures}

Human subjects: Consent was obtained by all participants in this study. University of Utah Institutional Review Board issued approval. Animal subjects: All authors have confirmed that this study did not involve animal subjects or tissue. Conflicts of interest: In compliance with the ICMJE uniform disclosure form, all authors declare the following: Payment/services info: All authors have declared that no financial support was received from any organization for the submitted work. Financial relationships: Erica F. Bisson declare(s) a grant from PCORI. Erica Bisson declare(s) stock/stock options from nView. Meic H. Schmidt declare(s) personal fees from Ulrich Medical USA. Andrew T. Dailey declare(s) a grant and personal fees from Biomet. Andrew T. Dailey declare(s) personal fees from AONA. Other relationships: All authors have declared that there are no other relationships or activities that could appear to have influenced the submitted work.

\section{Acknowledgements}

We thank Kristin Kraus, MSc, for editorial assistance with this paper.

\section{References}

1. Kim JY, Oh CH, Yoon SH, Park HC, Seo HS: Comparison of outcomes after atlantoaxial fusion with transarticular screws and screw-rod constructs. J Korean Neurosurg Soc. 2014, 55:25560. 10.3340/jkns.2014.55.5.255

2. Coyne TJ, Fehlings MG, Wallace MC, Bernstein M, Tator $\mathrm{CH}$ : C1-C2 posterior cervical fusion: 
long-term evaluation of results and efficacy. Neurosurgery. 1995, 37:688-92.

10.1227/00006123-199510000-00012

3. Elliott RE, Tanweer O, Boah A, Morsi A, Ma T, Frempong-Boadu A, Smith ML: Outcome comparison of atlantoaxial fusion with transarticular screws and screw-rod constructs: metaanalysis and review of literature. J Spinal Disord Tech. 2014, 27:11-28.

10.1097/BSD.0b013e318277da19

4. Lall R, Patel NJ, Resnick DK: A review of complications associated with craniocervical fusion surgery. Neurosurgery. 2010, 67:1396-402. 10.1227/NEU.0b013e3181f1ec73

5. Gruskay JA, Fu M, Bohl DD, Webb ML, Grauer JN: Factors affecting length of stay after elective posterior lumbar spine surgery: a multivariate analysis. Spine J. 2015, 15:1188-95.

10.1016/j.spinee.2013.10.022

6. Kehlet H, Wilmore DW: Multimodal strategies to improve surgical outcome. Am J Surg. 2002, 183:630-41. 10.1016/S0002-9610(02)00866-8

7. Kollef MH: Inadequate antimicrobial treatment: an important determinant of outcome for hospitalized patients. Clin Infect Dis. 2000, 31:131-38. 10.1086/314079

8. White RH, Romano PS, Zhou H, Rodrigo J, Bargar W: Incidence and time course of thromboembolic outcomes following total hip or knee arthroplasty. Arch Intern Med. 1998, 158:1525-31. 10.1001/archinte.158.14.1525

9. Keehan SP, Sisko AM, Truffer CJ, Poisal JA, Cuckler GA, Madison AJ, Lizonitz JM, Smith SD: National health spending projections through 2020: economic recovery and reform drive faster spending growth. Health Aff (Millwood). 2011, 30:1594-605. 10.1377/hlthaff.2011.0662

10. Eijkenaar F, Emmert M, Scheppach M, Schöffski O: Effects of pay for performance in health care: a systematic review of systematic reviews. Health Policy. 2013, 110:115-30.

10.1016/j.healthpol.2013.01.008

11. Basques BA, Bohl DD, Golinvaux NS, Gruskay JA, Grauer JN: Preoperative factors affecting length of stay after elective anterior cervical discectomy and fusion with and without corpectomy: a multivariate analysis of an academic center cohort. Spine (Phila Pa 1976). 2014, 39:939-46. 10.1097/BRS.0000000000000307

12. von Elm E, Altman DG, Egger M, Pocock SJ, Gotzsche PC, Vandenbroucke JP: The Strengthening the Reporting of Observational Studies in Epidemiology (STROBE) statement: guidelines for reporting observational studies. Ann Intern Med. 2007, 147:573-77. 10.1136/bmj.39335.541782.AD

13. Zheng F, Cammisa FP, Jr., Sandhu HS, Girardi FP, Khan SN: Factors predicting hospital stay, operative time, blood loss, and transfusion in patients undergoing revision posterior lumbar spine decompression, fusion, and segmental instrumentation. Spine (Phila Pa 1976). 2002, 27:818-24. 10.1097/00007632-200204150-00008

14. Bisson EF, Bowers CA, Hohmann SF, Schmidt MH: Smoking is associated with poorer qualitybased outcomes in patients hospitalized with spinal disease. Front Surg. 2015, 2:20. 10.3389/fsurg.2015.00020

15. Harrer SW, Carlson WO: Spinal health and smoking. S D Med. 2009, 62:311-13.

16. Lau D, Chou D, Ziewacz JE, Mummaneni PV: The effects of smoking on perioperative outcomes and pseudarthrosis following anterior cervical corpectomy: Clinical article. J Neurosurg Spine. 2014, 21:547-58. 10.3171/2014.6.SPINE13762

17. Turan A, Mascha EJ, Roberman D, Turner PL, You J, Kurz A, Sessler DI, Saager L: Smoking and perioperative outcomes. Anesthesiology. 2011, 114:837-46. 10.1097/ALN.0b013e318210f560

18. Glassman SD, Anagnost SC, Parker A, Burke D, Johnson JR, Dimar JR: The effect of cigarette smoking and smoking cessation on spinal fusion. Spine (Phila Pa 1976). 2000, 25:2608-2615. 10.1097/00007632-200010150-00011

19. Scivoletto G, Farchi S, Laurenza L, Tamburella F, Molinari M: Impact of multiple injuries on functional and neurological outcomes of patients with spinal cord injury. Scand J Trauma Resusc Emerg Med. 2013, 21:42. 10.1186/1757-7241-21-42

20. Putz C, Schuld C, Gantz S, Grieser T, Akbar M, Moradi B, Wiedenhofer B, Furstenberg CH, Gerner HJ, Rupp R: The effect of polytrauma as a possible confounder in the outcome of monotraumatic vs polytraumatic paraplegic patients: a clinical cohort study. Spinal Cord. 2011, 49:721-27. 10.1038/sc.2010.181

21. Smetana GW: Preoperative pulmonary evaluation. N Engl J Med. 1999, 340:937-44. 10.1056/NEJM199903253401207

22. Holte K, Klarskov B, Christensen DS, Lund C, Nielsen KG, Bie P, Kehlet H: Liberal versus 
restrictive fluid administration to improve recovery after laparoscopic cholecystectomy: a randomized, double-blind study. Ann Surg. 2004, 240:892-99.

10.1097/01.sla.0000143269.96649.3b

23. Pull ter Gunne AF, Skolasky RL, Ross H, van Laarhoven CJ, Cohen DB: Influence of perioperative resuscitation status on postoperative spine surgery complications. Spine J. 2010, 10:129-35. 10.1016/j.spinee.2009.10.002

24. Kamath AF, Austin DC, Derman PB, Israelite CL: Unplanned hip arthroplasty imposes clinical and cost burdens on treating institutions. Clin Orthop Relat Res. 2013, 471:4012-19.

10.1007/s11999-013-3226-X

25. Taggard DA, Kraut MA, Clark CR, Traynelis VC: Case-control study comparing the efficacy of surgical techniques for C1-C2 arthrodesis. J Spinal Disord Tech. 2004, 17:189-94.

10.1097/00024720-200406000-00005

26. Gluf WM, Brockmeyer DL: Atlantoaxial transarticular screw fixation: a review of surgical indications, fusion rate, complications, and lessons learned in 67 pediatric patients. J

Neurosurg Spine. 2005, 2:164-69. 10.3171/spi.2005.2.2.0164

27. Neo M, Fujibayashi S, Miyata M, Takemoto M, Nakamura T: Vertebral artery injury during cervical spine surgery: a survey of more than 5600 operations. Spine (Phila Pa 1976). 2008, 33:779-85. 10.1097/BRS.0b013e31816957a7

28. Ahmad FU, Wang MY: Lateral mass of C1 fixation and ponticulus-posticus . World Neurosurg. 2014, 82:e145-46. 10.1016/j.wneu.2014.02.011

29. Neo M: An essential principle for safe C1-2 transarticular screw insertion . J Spinal Disord Tech. 2008, 21:76-77. 10.1097/BSD.0b013e318163571e

30. McCarthy I, Hostin R, O'Brien M, Saigal R, Ames CP: Health economic analysis of adult deformity surgery. Neurosurg Clin N Am. 2013, 24:293-304. 10.1016/j.nec.2012.12.005 